\title{
Octavio Paz: Entre Modernidad e identidad
}

\section{Octavio Paz: Between Modernity and Identity}

\author{
Oliver Kozlarek ${ }^{1}$
}

\author{
Universidad Michoacana de San Nicolás de Hidalgo \\ (México) y Universität Vechta (Alemania)
}

Recibido: 06-04-20

Aprobado: 21-04-20

\section{Resumen}

Los debates en teoría social sobre la modernidad se han distanciado de la idea de convergencia de la que partían las "teorías de la modernización", desarrollando una conciencia de diferencia más sofisticada que no deja de interesarse por las coincidencias y afinidades globales y universales de las "múltiples experiencias" con y en la modernidad global. En el contexto de estos debates se constata que los procesos de modernización son entendidos como dinámicas que reciben su energía de dos fuerzas que, más que antagónicas, se complementan y se pueden resumir en los conceptos "modernidad" e "identidad". Este par de conceptos ha orientado importantes debates políticos y académicos en América Latina. Discutiré el ejemplo de Octavio Paz proponiendo que sus ideas en torno a la modernidad se orientan por los siguientes ejes temáticos: la condition moderne, la conditio humana y la condición mexicana.

Palabras-clave: "conciencia del mundo", identidad, modernidad, Octavio Paz, Teorías de la modernidad.

\footnotetext{
${ }^{1}$ (okozlarek@yahoo.com ) Profesor e investigador en la Universidad Michoacana de San Nicolás de Hidalgo. Actualmente es investigador asociado en la Facultad III de la Universidad de Vechta en Alemania donde también es coordinador científico del recién creado Kulturanthropologisches Institut Oldenburger Münsterland. Entre sus publicaciones recientes destacan: Moderne als Weltbewusstsein. Ideen für eine humanistische Sozialtheorie in globalen Moderne, Bielefeld, Transcript, 2011 (traducción: Modernidad como conciencia del mundo. Ideas para una teoría social humanista en la modernidad global, Siglo XXI, 2014); con Jörn Rüsen y Ernst Wolff: Shaping a Human World. Civilizations - Axial Times - Modernities - Humanisms, Bielefeld, 2012; Multiple Experiencies of Modernity, Göttingen: Vandenhoek \& Ruprecht, 2014; Modernidad, critica y humanismo, Morelia: UMSNH. 2015; Postcolonial Reconstruction. A Sociological Reading of Octavio Paz: Springer, 2016. Actualmente trabaja en un libro sobre un posible "Humanismo crítico".

ORCID: https://orcid.org/0000-0003-4971-6918.
} 


\begin{abstract}
The debates in social theory on modernity have clearly distanced from the idea of convergence from which the "theories of modernization" still started, developing a more sophisticated awareness of difference that does not cease to be interested in the global and universal coincidences and affinities of the "multiple experiences" with and within global modernity. In the context of these debates the processes of modernization are understood as dynamics that receive their energy from two forces that more than antagonistic complement each other and that can be summarized in the concepts "modernity" and "identity". This pair of concepts has been guiding important political and academic debates in Latin America. I will discuss the example of Octavio Paz by proposing that his ideas on modernity are oriented by the following thematic axes: the condition moderne, the conditio humana and the condición mexicana.
\end{abstract}

Key-words: "World Consciousness", Identity, Octavio Paz, Theories of Modernity.

\title{
Modernidad e identidad - desde la teoría social
}

1. De acuerdo con las teorías sociales clásicas, "modernización" se refiere a una lucha sistemática en contra de la tradición. "Modernidad" y "tradición" son vistas como fuerzas antagónicas: donde rige la tradición no hay modernidad y viceversa (Lerner 1958). La resistencia en contra de los proyectos modernizadores se apoyaba y se sigue apoyando muchas veces en estrategias que se arraigan en políticas de la identidad que muchas veces confunden identidad con tradición. También desde esta perspectiva "modernización" es vista como un proceso que neutraliza identidades colectivas. Sin embargo, en el interior de los debates sobre modernidad desde los años 1960 estas ideas empezaban a ser cuestionadas seriamente. El sociólogo Shmuel N. Eisenstadt observaba que las sociedades en proceso de modernización manifiestan una realidad diferente: los procesos de la modernización ocurren más bien en y a través de las respectivas tradiciones (Eisenstadt 1973). Eisenstadt es también el autor principal de un paradigma nuevo en la investigación sobre la modernidad que culmina en la idea de que pudieran existir "modernidades múltiples" (véase: Eisenstadt 2000) y que las diferencias se arraigan en las respectivas particularidades culturales. Modernidad e identidad ya no se oponen, sino empiezan a formar una formación conceptual dialéctica y productiva.

2. Recordando los debates más recientes en torno a la modernidad y los procesos de modernización también resalta otra particularidad. No solamente se multiplican las narrativas y las respectivas formas de vida modernas, sino 
que también se introduce de manera cada vez más visible el reconocimiento del entrelazamiento de los asuntos humanos a nivel planetario. Ya en los años 1980 el historiador J. G. A. Pocock mantuvo la idea de que modernidad es más que nada una conciencia específica (véase: Pocock 1987). En un libro que publiqué hace unos años parto de la idea de que una de las especificidades de esta conciencia es la de entender justamente el entrelazamiento de los asuntos humanos a nivel planetario. Dicho de otra manera: modernidad es también una suerte de "conciencia del Mundo" (véase Kozlarek 2014a).

Más aún: es posible argumentar que esta conciencia del mundo se manifiesta también en y a través de varios de los debates actuales en las ciencias sociales y las humanidades como el posmodernismo, la crítica postcolonial y más evidentemente el debate en torno a la globalización (véase: Kozlarek 2014a).

Ahora bien, la multiplicación de modernidad, por un lado, y el reconocimiento del entrelazamiento planetario, por el otro, reanimaron también los debates en torno a la "convergencia" de los procesos de modernización (véase: Schmidt 2014). A un nivel más abstracto esto significa: el tema que surge nuevamente como problema es la relación entre lo particular y lo universal. Modernidad aparece entonces ya no como una condición que elimina las particularidades de las sociedades, sino más bien como una condición en la cual éstas son pensadas en relación con las afinidades y coincidencias. La teoría de la modernidad se ha transformado, entonces, desde una teoría de convergencia y de universalización en una teoría con una conciencia de diferencia significativa que, sin embargo, no deja de preocuparse por las tendencias generalizadoras y las orientaciones normativas universales de las transformaciones sociales, políticas y culturales a nivel planetario.

3. Finalmente, creo que las múltiples narrativas en torno a la modernidad pueden ocupar un lugar privilegiado en la actualidad. A través de estas prácticas narrativas se expresan, se enfrentan y se traslapan ofertas de sentido que ayudan a comprender tanto los procesos históricos como los retos del presente y para el futuro. "Modernidad", en esta comprensión, deja de ser un concepto formativo de la sociología y se convierte en un campo cultural en el cual se desdoblan narrativas que permiten renegociar las particularidades de los respectivos grupos sociales al exponerlos a las exigencias y los retos globales (véase: Wagner 2001; 2008). O, dicho de otra manera: donde se renegocian visiones del mundo y de lo humano entre particularidades identitarias y exigencias de una modernidad global. Las dinámicas que estos procesos narrativos manifiestan producen antagonismos y conflictos y se energetizan en un campo de tensión marcado por los conceptos "modernidad" e "identidad".

En lo que sigue quisiera mostrar que en el pensamiento de Octavio Paz se arraiga un compromiso serio con la modernidad, no solamente en una conciencia 
del mundo, sino también en el reconocimiento de exigencias históricas que afectan a todas las sociedades del planeta. De tal manera que en el pensamiento de Paz "modernidad" no se opone a tradición, sino que forma más bien una pareja dialéctica con "identidad".

\section{Modernidad e identidad en el pensamiento latinoamericano}

En un artículo reciente, el sociólogo Sérgio Costa constata que en América Latina la modernidad siempre aparecía desde una distancia tanto temporal como espacial, ya que “[t]he term 'modern' in Latin America commonly involves a spatial and temporal association of modernity with Western Europe, later with North America as well" (Costa 2019: 839). Para darle más peso a esta idea Costa se apoya justamente en Octavio Paz citándolo con las siguientes palabras de su conferencia de aceptación del Premio Nobel en 1990: "This is why there was frequent talk of "Europeanizing" our countries: the modern was outside and had to be imported" (cit. en: íbid.) $)^{2}$.

Más que una amenaza a la tradición, "modernidad" es vista, entonces, como algo extraño, en fin: como una amenaza a lo propio, a la mismidad. Este problema acecha sobre todo a las sociedades postcoloniales ${ }^{3}$. Pero en América Latina la independencia oficial ocurrió ya hace más o menos 200 años, y se puede observar la articulación de un pensamiento que maduró precisamente en el campo de la tensión entre modernidad e identidad.

Esta posición "entre" o "dialéctica" marca significativamente no solamente a los pensadores latinoamericanos, sino también a las dinámicas históricas y políticas como revela el sociólogo chileno Jorge Larraín en un libro destacable cuyo objetivo el autor resume en las siguientes palabras:

The objective of this book is to show historically how, within the context of some distinct stages, Latin America has been simultaneously constructing its cultural identity and modernizing, and the way in which these two phenomena, in spite of being intimately interconnected, are frequently perceived as opposite alternatives (Larraín 2000: 6).

La cita deja clara que para Larraín modernidad e identidad no se oponen. Sin embargo, él reconoce también que muchas veces son vistas como orientaciones excluyentes. Lo anterior lo explica Larraín justamente con el hecho de que la modernidad es entendida como imposición externa:

\footnotetext{
2 Véase también: Vuelta, núm. 170 (enero 1991), 10-14.

${ }^{3}$ Laura Carballido Coria, ha demostrado recientemente que esto es cierto también para la India (véase: Carballido 2018).
} 
[...] this opposition between modernity and identity is also a consequence of the fact that modernity in Latin America has been often conceived as a process intrinsically connected with the trajectory of Western societies (Europe and the United States) in which a particular set of institutions allowed the development of a market economy, a democratic polity and an autonomous scientific knowledge. Instead of conceiving of modernity as a way of dealing with basic problematics of social life which seek autonomy in the political and epistemic fields and rational control in order to satisfy basic needs, it confuses the particular institutional answer of Western societies with a general and necessary feature of modernity (ibid.).

La historiadora británica Nicola Miller percibe el mismo problema. Pero ella también reconoce que las tensiones producidas en el campo de fuerzas que se extiende entre los polos conceptuales de "modernidad" e "identidad" pueden suscitar actitudes diferentes. La primera se centra en una aceptación absolutamente acrítica de las dinámicas "tecnocráticas" y "eurocéntricas" de la modernización. Es decir: la heteronomía y excentricidad de la modernidad desde una perspectiva latinoamericana es asumida y considerada como un aspecto positivo e incluso deseable. La segunda respuesta tiende hacia el polo opositor, a saber: en ella se agudiza un rechazo igualmente total de modernidad como algo externo desde una posición fincada en una suerte de identidad esencialista.

Pero de acuerdo con Miller, existe también una tercera actitud cultivada y representada por un grupo importante de intelectuales en América Latina y que no se reduce ni a la aceptación ni al rechazo absolutos, sino que se condensa en "visiones" o "imaginarios" de modernidad sumamente creativos y originales (véase Miller 2008). En su libro Miller trata de mostrar que estas "modernidades latinoamericanas" ya se articularon con mucha claridad en las primeras tres décadas del siglo XX. Podemos decir entonces que una conciencia moderna ya existía en América Latina mucho antes de que las "teorías de la modernización" empezaran a recetar la modernización en la región después de la Segunda Guerra Mundial.

Pero también podemos observar que, de acuerdo con estas otras modernidades, la tensión conceptual entre "modernidad" e "identidad" ha generado proyectos intelectuales e históricos muy sugerentes. Desde una perspectiva de la historia de las ideas, Miller recupera sobre todo las ideas de algunos de los intelectuales latinoamericanos de las primeras décadas del siglo XX más destacados: José Enrique Rodó, Juan B. Busto, Alfonso Reyes y José Carlos Mariátegui. Los retos que se asocian con la idea de la modernidad están presentes en todos estos autores. Pero las soluciones requieren, según ellos, también de una conciencia de las diferencias locales y regionales. Parto aquí de la convicción de que el pensamiento de Octavio Paz es digno de ser integrado en esta línea de intelectuales latinoamericanos. A pesar de 
que él pertenece claramente a una generación posterior, sus ideas manifiestan inquietudes parecidas: también en la obra de Paz se puede vislumbrar una clara preocupación por una modernidad con identidad mexicana en un contexto inevitablemente global.

\section{Los tres ejes de la modernidad en la obra de Octavio Paz}

Como he tratado de mostrar en otra publicación (Kozlarek 2014a), el tejido de ideas que estructuran los trabajos ensayísticos de Octavio Paz en torno a la modernidad adquieren su solidez a través de tres ejes fundamentales: la reflexión sobre la modernidad misma (la condition moderne), el reconocimiento de la necesidad de asegurarse de un anclaje normativo en una antropología y un humanismo (crítico) (la conditio humana) y, finalmente, el análisis cultural sobre cómo modernidad e identidad se entrelazan en el caso de México (la condición mexicana).

\section{a) La condition moderne}

En un escrito autobiográfico Paz dice:

La Edad Moderna, desde el Renacimiento, ha sido la de la ruptura: hace ya más de quinientos años que vivimos la discordia entre las ideas y las creencias, la filosofía y la tradición, la ciencia y la fe. La modernidad es el período de la escisión [...] Nuestro tiempo es el de la conciencia escindida y el de la conciencia de la escisión. Somos almas divididas en una sociedad dividida. (Paz 1993: 43; cursivas en el original).

Sin embargo, este diagnóstico no lo motiva a resignarse. Rechazando tanto el nihilismo como el cinismo Paz asumía esta comprensión de la modernidad más bien como un reto permanente ante el cual una cultura se debe instalar en los intersticios entre modernidad e identidad.

Paz no entendía modernidad exclusivamente desde la perspectiva de los debates teórico-sociales. Más bien la veía como un reto permanente tanto para su país México como para su propia biografía. "La búsqueda de la modernidad democrática ha sido en la historia de México un problema y una constante y, en la biografía intelectual de Octavio Paz, casi un destino", escribe también Ricardo Pozas Horcasitas y continua:

[e]sta búsqueda fue uno de los objetivos que dio sentido e identidad a su acción pública y fue uno de los ejes analíticos con el que explicó y dio sustento a sus posturas políticas, concepción teórica y principio de acción que atravesaron su carrera intelectual (Pozas Horcasitas 2009: 246). 
El compromiso con la modernidad es también consecuencia de que Paz no pensaba que lo moderno viene de Europa o de EEUU y que México debe asumirlo de manera acrítica. No lo veía como una imposición que viene de fuera. Más bien se trató para él de un reto que se impone a todas las sociedades actuales. Haciendo eco de una idea ya expuesta en El laberinto de la soledad, Paz reafirma en 1993:

La suerte de México no es distinta a la del mundo; la pregunta sobre la modernidad y su desenlace en el siglo XXI también nos concierne a nosotros. Me atreví a decirlo hace ya más de cincuenta años: "por primera vez en la historia, somos contemporáneos de todos los hombres". (Paz 1993: 41-42).

Entendiendo a la modernidad como un reto que comparten todas las sociedades, expresa con claridad la "conciencia del mundo" propia de la concepción de modernidad que encontramos en la obra de Paz. Sin embargo, "modernización" no resume solamente un conjunto de cambios sociales formales tales como "racionalización", "burocratización", "democratización", "urbanización", etc. Tampoco se trata de un proceso económico en el cual se consolida el dictado del mercado como realidad global. Más bien se trata también de una dinámica cultural que se plasma en la conciencia de que los asuntos humanos están incuestionablemente entrelazados a nivel planetario. Puede ser que esta conciencia se haya articulado de manera más clara en y a través del debate sobre la globalización a partir de 1990. Pero, hay buenas razones para pensar que algo así como la "conciencia moderna" siempre contemplaba también -si no en primer lugar- una suerte de conciencia del mundo (véase Kozlarek 2014a).

Pienso no solamente que la obra de Octavio Paz representa una expresión muy elocuente de esta "conciencia del mundo", sino que ésta ocupa un lugar central en la arquitectura de sus ideas. Esto se plasma sobre todo en El laberinto de la soledad, no obstante que muchos encuentran el centro de gravedad de esta obra en la idea de la identidad. Así escribió por ejemplo el historiador David Brading hace algunos años:

El postulado central de este estudio era la existencia del 'ser' mexicano, encarnizado en la nación con la que él [Octavio Paz, O.K.] se identificaba con todo el fervor, evocando en todo momento el 'nosotros colectivo' cuyo viaje mental quería cartografiar a través de los tiempos. (Brading 2002: 37-38).

Con esta evaluación Brading sitúa su interpretación del libro sin lugar a dudas en la tradición de quienes consideran El laberinto como una contribución al gran número de obras que buscaban describir la "esencia", la "ontología", 
la "psicología", el "carácter", la "condición" e incluso la "sexualidad" de los mexicanos 4 . Pero Paz mismo asegura con toda claridad que su "libro no es un ensayo sobre una quimérica 'filosofía del mexicano'; tampoco una descripción psicológica ni un retrato", y continúa explicando que se trata más bien de un análisis "[...] de nuestra situación en el mundo moderno" (Paz 1992: 25).

Se trata entonces con toda intención de un libro sobre la modernidad o, mejor dicho: de una suerte de análisis o "crítica cultural" de la "experiencia de la modernidad" de la cual el propio formato de esta experiencia entra de manera autobiográfica como un aspecto del análisis importante (véase: Stanton 2015: 199 ss.). Esta decisión para ocuparse del tema de la modernidad ya crea una enorme distancia con los intentos de las ciencias sociales de lidiar con el tema de la modernidad y sobre todo con los procesos de cambio para los cuales se acuñaba el término de la modernización. Mientras estos intentos después de la Segunda Guerra Mundial proclamaron que sociedades como la mexicana aún no habían llegado a la modernidad, las experiencias que Paz relata afirman una modernidad mexicana incuestionable.

En este sentido El laberinto de la soledad pone de manifiesto lo que Marshall Berman expresa unas tres décadas más tarde:

There is a mode of vital experience -experience of space and time, of the self and others, of life's possibilities and perils- that is shared by men and women all over the world today. I will call this body of experiences "modernity". (Berman 1982: 15).

Tal como lo vemos en el libro de Berman, modernidad no es, entonces, la visión de un mundo unido venidero, sino una realidad ya establecida que se revela en y a través de las experiencias de las personas. La pregunta no es si queremos o no ser modernos, sino cómo nos instalamos en la condición global de la modernidad o, dicho de un modo un poco más derrotista: ¿cómo lidiar con ella? Modernidad como reto y búsqueda en el sentido de Paz obliga también a realizar este tipo de preguntas.

Podemos decir también: lo que nos une en la modernidad no es una meta que algunos han alcanzado y otros no, sino una serie de experiencias que hacemos todos (véase: Kozlarek 2014b). Esto lo sabía Paz, y Berman lo expresó más tarde así: "Modern environments and experiences cut across all boundaries of geography and ethnicity, of class and nationality, of religion and ideology: in this sense, modernity can be said to unite all mankind" (Berman 1982: 15).

\footnotetext{
${ }^{4}$ Estas palabras clave proceden de una antología sobre este tema que Roger Bartra publicó hace algunos años (Bartra 2002).

Araucaria. Revista Iberoamericana de Filosofia, Política, Humanidades y Relaciones Internacionales, año $22, \mathrm{n}^{\circ} 43$. Primer semestre de 2020. Pp. 251-266. ISSN 1575-6823 e-ISSN 2340-2199 https://dx.doi.org/10.12795/araucaria.2020.i43.12
} 


\section{b) La conditio humana}

Liliana Weinberg señaló en su contribución a una antología sobre el humanismo en México que Paz combina la crítica de la modernidad con un "humanismo crítico".

En el caso de Paz [...] es importante adelantar que desde la propia obra se advierte la presencia de un elemento existencial fundamental -que puede también rastrearse en sus propias evocaciones de infancia-, y es la intuición primera de un desgarramiento hombre-mundo, de una separación entre el individuo y su comunidad de origen, que él luego extenderá a la condición humana toda y rearticulará en la dialéctica soledad-comunión, verdadera matriz de su pensamiento, y como constante búsqueda de reconciliación, de restauración de ese orden originario perdido, a través de la fiesta, el mito, la poesía, el erotismo, la revuelta. He aquí, insisto, una matriz fundamental para acercarnos a Paz (Weinberg 2004: 373).

La centralidad que Paz asigna al tema del "desgarramiento hombre-mundo" justifica una suerte de "humanismo crítico" no en última instancia pues revela como profundamente arraigado en la experiencia humana un problema para el cual los seres humanos no tenemos una solución definitiva. Si modernidad es sobre todo reto y búsqueda, lo podemos entender claramente en este contexto.

Aunque Paz no entiende este problema como un problema exclusivamente moderno, tampoco piensa que la apuesta moderna por la razón represente una solución. Esto se expresa, por ejemplo, en la manera en la cual él descalifica a la filosofía como una herramienta adecuada. La filosofía no solamente fracasa como herramienta para resolver el dilema humano, sino en cuanto antropología filosófica se limita a una función meramente sintomática: ella surge en momentos en los cuales la duda en lo humano se agudiza. A todo ello le subyace la idea de que "[...] la visión del filósofo es un todo en el que faltan muchas cosas" (Paz 1996: 527).

Pero el humanismo de Paz también merece el adjetivo "crítico" por otra razón: Paz parece haber considerado que las estrategias para superar el sentimiento del desgarramiento no solamente son necesarias, sino que también están condicionadas al permanente fracaso. ¿Cuáles son estas estrategias? Habrá que mencionar aquí todas las construcciones a través de las cuales los seres humanos tratamos de regular nuestras relaciones con la naturaleza y con los demás seres humanos y que desembocan en las creaciones humanas más importantes: los mitos, los rituales y las fiestas, las religiones o, en términos más generales: las culturas, las sociedades y las civilizaciones. En este sentido todas estas formaciones colectivas y simbólicas narran, en realidad, una sola historia: la de la soledad de los seres humanos -y la tienen que narrar una y otra vez, ya que ninguna de estas historias puede ser definitiva-. 
Aquí se abrirían caminos de investigación muy interesantes: podemos preguntar, por ejemplo, cómo las diferentes culturas cumplen con esta función, y también -desde una perspectiva más crítica- cómo fracasan en estos intentos. En El laberinto de la soledad Paz da algunos pasos en esta dirección cuando compara la sociedad y la cultura norteamericana y la mexicana. Y El laberinto constituye obviamente una reflexión sobre México en estos términos. En este sentido El Laberinto es mucho más un intento de crítica cultural orientada en la antropología de la soledad que un libro sobre la identidad cultural o nacional de los mexicanos.

Pero Paz pretende algo más: la verdadera condición humana no está en las propuestas para resolver la experiencia del desgarramiento, de la separación o de la soledad; no está en la cultura, en la sociedad o en la civilización, sino en la capacidad de hacer esta situación consciente. Esto es posible gracias a la experiencia poética. La experiencia poética significa dos cosas: por una parte, refleja la conciencia del entrelazamiento de todo con todo. Así escribe Paz en una carta a Roger Caillois: "A mi juicio, la experiencia de usted se acerca más a lo que se llama la 'experiencia poética' y que consiste en ver al mundo como un sistema de correspondencias, un tejido de acordes" (Paz 1994b: 468).

Por otra parte, la experiencia poética manifiesta que las respuestas definitivas a las experiencias de la soledad no pueden ser definitivas ya que siempre queda un resto que se resiste a integrarse en las construcciones sociales y culturales. En este sentido, en la experiencia poética resalta siempre lo disidente, lo oculto, lo otro (véase: Weinberg 2004: 377). Aquí las ideas de Paz se asemejan a las del Collège de Sociologie -que ha sido una influencia importante para Paz en el momento de escribir El laberinto-, que el sociólogo alemán Stephan Moebius ha definido como la "ciencia de lo no asimilable, del resto oculto y de lo marginado de la razón y del orden homogéneo" (Moebius 2009: 16). El propio Paz lo expresa así: "Afirmo que la poesía es irreductible a las ideas y a los sistemas. Es la otra voz. No la palabra de la historia ni de la antihistoria, sino la voz que en la historia dice siempre otra cosa, la misma desde el principio" (Paz 1971: 109).

\section{c) La condición mexicana}

La gran pregunta para Paz era entonces: “¿cómo crear una sociedad, una cultura, que no niegue nuestra humanidad, pero tampoco la convierta en una vana abstracción?" (Paz 1998: 91). Paz sabía que esta pregunta no se ha planteado únicamente a los mexicanos sino a todos los seres humanos que están unidos en la modernidad global. No obstante, la pregunta por la conditio humana no sólo está vinculada inseparablemente a la pregunta por la condition moderne, sino también con la pregunta por las condiciones específicas de cada 
sociedad. Este es el gozne que da cohesión a las diferentes dimensiones de experiencia en la modernidad global y que ha de proveer orientación en el "laberinto de la soledad".

Como ya habíamos visto, la pregunta por lo mexicano no seduce a Paz a retomar los hilos de los debates sobre la identidad nacional y cultural en los cuales se involucraron los intelectuales y los académicos mexicanos después de la revolución. Más bien la ve vinculada intrínsecamente con la pregunta por la humanidad. Susanne Klengel culmina un capítulo sobre Paz con las siguientes palabras:

Estas conclusiones arrojan nueva luz sobre el llamamiento humanista a la responsabilidad del individuo en la historia de la humanidad e iluminan su intenso alegato en El laberinto de la soledad por el reconocimiento de la 'otredad' y por la comunicación y el diálogo, y su proscripción de toda forma de exclusión y discriminación. La contribución de Paz al discurso sobre la identidad mexicana es, por lo tanto, reconocible como una forma de pensar que sólo podría concebir su propia identidad en relación con un marco de referencia humano más amplio (Klengel 2011: 252).

Pero para realmente entender la particularidad con la cual Paz se refiere al debate sobre la identidad mexicana, vale la pena enfocarnos en algunas decisiones teóricas y conceptuales. Con este objetivo me permito referirme brevemente a una observación importante: en contra del prejuicio de que las ciencias sociales y las humanidades no aportarían nada útil a las sociedades, los sociólogos mexicanos Óscar F. Contreras y Cristina Puga coordinaron hace poco un libro colectivo muy sugerente. Bajo el título Las ciencias sociales y el Estado nacional en México juntaron algunos trabajos que tratan de mostrar que

[d]esde el inicio de la historia independiente de México, y particularmente a partir del nacimiento de la República Federal en 1824, las entonces incipientes ciencias sociales $[\ldots]$ sentaron las bases sobre las que hoy descansa buena parte del entramado social, institucional, político, económico y cultural del país (Contreras/Puga 2018: 9).

Un aspecto importante es, en este sentido, la labor de los intelectuales y de los académicos que "forjaron una patria" después de 1910. Ahora bien: de acuerdo con Aurora Gómez Galvarriato, en este contexto habrá que mencionar sobre todo a tres: Andrés Molina Enríquez (1868-1940), Manuel Gamio (18831960) y José Vasconcelos (1882-1959). Cabe mencionar que los tres se destacan por la voluntad de distanciarse significativamente de las doctrinas -por cierto: también social-científicas- del positivismo y del evolucionismo social, sobre las cuales se erigían las estructuras ideológicas del Porfiriato. Pero, como bien demuestra Gómez Galvarriato en su trabajo, los autores mencionados también 
tienden a reproducir algunas presuposiciones. Ella se refiere sobre todo al hecho de que la discusión de las bases de lo social retoma de manera muy poco crítica las presuposiciones de las doctrinas raciales que estaban de moda en el pensamiento político y social en esos tiempos. Esto ciertamente es más correcto en el caso de Andrés Molina Enríquez y José Vasconcelos y menos en el caso de Manuel Gamio quien, bajo la influencia de los antropólogos culturales Eduard Seler y Franz Boas, desarrollaba nociones más culturales que raciales, pero igualmente esencialistas.

Ahora bien: Paz rompe totalmente con estas tendencias. Él se distancia de cualquier esencialismo, sea este racial o cultural. Como ya lo habíamos dicho: El laberinto de la soledad representa más bien una suerte de crítica cultural de la sociedad mexicana en la cual la cultura no se reduce a la función de codificar identidades esenciales. Más bien aparece en el libro como un tejido de significados que se modifica permanentemente en los interminables movimientos por resolver los problemas humanos, sobre todo el de la experiencia de la soledad.

A Paz le interesa esta creación de los significados culturales y él debe haber pensado que estos procesos son mucho más creativos de lo que aquellos que se interesan por la identidad sugieren una y otra vez. De hecho, podemos leer El laberinto de la soledad también como un intento de reorientar a la cultura mexicana a través de una interpretación nueva y creativa.

Una y otra vez se ha comentado que Paz habría dibujado una imagen desafortunada de México. Algunas de las categorías que Paz coloca en el centro de sus análisis pueden, en efecto, dar la impresión de una visión negativa de este país, su cultura y su gente, empezando, por su puesto, con el diagnóstico de la soledad. Sin embargo, pienso que estos juicios son equivocados. Paz era un intelectual crítico, pero su objetivo no fue desacreditar a su país. Antes bien, procuraba "intervenir" en el tejido de significados culturales, proponiendo interpretaciones nuevas. Es en este sentido como Paz entendía su tarea de intelectual. Quisiera mencionar aquí tan sólo dos ejemplos que motivaban a Paz en los años 40 y que se expresan con toda claridad en El laberinto de la soledad:

1. Para Paz existen tres figuras femeninas que él estiliza como pilares de la cultura mexicana: La Virgen de Guadalupe, que simboliza el sincretismo religioso y cultural, la "Malinche", que también se entiende como símbolo del mestizaje cultural, y la poetisa y clériga Sor Juana Inés de la Cruz, en la que Paz parece haber descubierto nada más y nada menos que el prototipo de la intelectualidad mexicana.

$\mathrm{Al}$ colocar estas tres figuras en el centro de su interpretación de la cultura mexicana, Paz presenta ahora a sus conciudadanos una imagen de la cultura mexicana que se aleja consciente y explícitamente de ideas generadas por 
otros autores como Samuel Ramos, quien pone en el centro de su análisis de la mexicanidad el "pelado" (Ramos 1999 [1934]) -un personaje que se destaca por sus formas rudas y poco solidarias en el trato de otras personas y quien condensa al machismo--. Aunque Paz no niega el machismo, demuestra cuáles alternativas se pueden extraer de la cultura mexicana.

Estas intervenciones virtuosas en la cultura, que no sólo cuestionan los supuestos inequívocos, sino que también los reinterpretan de manera creativa, representan una especie de crítica cultural proactiva que naturalmente va más allá de las exigencias de las cuestiones estéticas del gusto y que parecen capaces de abordar problemas sociales muy concretos. La visión de Paz podría resumirse de la siguiente manera: los problemas sociales y políticos también tienen raíces culturales. Pero no se trata de hablar de un determinismo cultural, porque las culturas pueden ser reinterpretadas y, por lo tanto, la acción política y social que es posible realizar a través de ellas puede ser recodificada. $\mathrm{O}$, dicho en las palabras del sociólogo Jeffrey C. Alexander: "If the world is itself based on collective understandings, then changing the world always involves, in some large part, changing these understandings in turn" (Alexander 2003: 193).

2. Otro tema que para Paz ha sido importante -y que igualmente se expresa de manera muy clara en El laberinto de la soledad- es el de la modernidad entendida como "conciencia del mundo". Como ya habíamos dicho: Paz no estaba realmente interesado en fijar algo así como la identidad cultural mexicana, sino en recodificar la cultura mexicana, pero esto lo quería hacer justamente porque pensaba a México como un país que formaba parte de un entrelazamiento global de países.

El tejido moderno global de los asuntos humanos era para Paz una realidad en la cual México participa y debe participar no solamente en un sentido intelectual sino también en lo político. Es de esta manera en la que se deben entender las siguientes palabras:

Termino estas reflexiones: los cambios de México corresponden, aproximadamente, a los del mundo. Aunque el proyecto de modernización viene del gobierno, ha sido la respuesta a una demanda colectiva de cambio, muchas veces implícita, como todo lo que brota del fondo social. La participación popular no tiene por qué traducirse en asentimiento mecánico; puede manifestarse como diálogo, crítica o divergencia. La modernización no busca sólo partidarios: también busca interlocutores (Paz 1990: 78).

Paz aboga aquí por una modernización diferenciada que no solamente busque repetir y reproducir lo que viene del exterior, sino en y a través de la cual el país se vincula con el mundo de manera más afirmativa, rompiendo de una vez para siempre con el patriotismo tradicionalista que dominaba a la política mexicana en momentos anteriores: "Modernizar no es copiar sino adaptar; 
injertar y no transplantar. Es una operación creadora, hecha de conservación, imitación e invención" (ibid. 57-58).

En los años 40 Paz vislumbra un reto importante y lo plantea con toda claridad: la pregunta que debe motivar la búsqueda de los pueblos, de las naciones y de los países ya no es la pregunta por la identidad, sino la pregunta por la modernidad; y ésta es también la pregunta por la constelación global de los asuntos humanos.

\section{Conclusión}

En la obra de Paz -y sobre todo en El laberinto de la soledad-encontramos un ejemplo muy lúcido de un "imaginario social de la modernidad" (Taylor 2004) que no solamente asume una clara "conciencia del mundo", es decir: el reto de que los asuntos humanos están irrevocablemente entrelazados a nivel planetario, sino que reconoce también que las herramientas culturales necesarias para enfrentar a esta situación siguen siendo diferentes. De tal manera que resolver los problemas que la modernidad global suscita, requiere siempre de estrategias que deben ser resultados de un cabildeo ingenioso entre modernidad e identidad.

La actualidad de estas ideas que Paz expone en su obra es evidente. Por un lado, queda claro que una concepción de modernidad sin identidad está condenada a convertirse en un proyecto demasiado abstracto. Pero, por otro lado, podemos observar que los excesos de una política de la identidad que se desentiende de los asuntos humanos, más allá de los confines de su propia cultura, del propio pueblo, etc., no logra asumir los retos que hoy todos los seres humanos compartimos.

\section{Referencias bibliográficas:}

Alexander, Jeffrey C. (2003). The Meanings of Social Life: A Cultural Sociology, Oxford/New York, et al.: Oxford University Press.

Bartra, Roger (Ed.) (2002). Anatomía del mexicano, Ciudad de México: Plaza y Janés.

Berman, Marshall. (1982). All that is solid melts into air/The experience of modernity, Nueva York, Simon \& Schuster.

Brading, David A. (2002). Octavio Paz y la poética de la historia mexicana, Mexico, D.F.: Fondo de Cultura Económica. 
Carballido Coria, Laura. (2018). "El proyecto de la modernización de Dehli", en: Akuavi Adonon Viveiros/Laura Carballido Coria/Jorge Galindo/ Fernanda Vázquez Vela (2018). Modernización y espacio. Imaginarios, Ordenamientos y prácticas, Ciudad de México: Universidad Autónoma Metropolitana/Universidad del Rosario, 215-243.

Contreras, Óscar/Cristina Puga (Eds.) (2018). Las ciencias sociales y el Estado nacional en México, Ciudad de México: Fondo de Cultura Económica.

Costa, Sergio. (2019). "The Research on Modernity in Latin America: Lineages and Dilemmas", en: Current Sociology, Vol. 67(6), 838-855.

Eisenstadt, Shmuel N. (1973). Tradition, Wandel und Modernität, Frankfurt/M.: Suhrkamp.

Eisenstadt, Shmuel N. (2000). ,Multiple Modernities”, en Shmuel N. Eisenstadt. et al. (Eds.), Daedalus, 129, 1-29.

Gómez Galvarriato, Aurora: "La revolución de 1910, las identidades originarias, la fundación del nuevo nacionalismo y el mito de la identidad nacional", en: Contreras, Óscar/Cristina Puga (Eds.) (2018). Las ciencias sociales y el Estado nacional en México, Ciudad de México: Fondo de Cultura Económica,

Klengel, Susanne. (2011). Die Rückeroberung der Kultur: lateinamerikanische Intellektuelle und das Europa der Nachkriegsjahre (1945-1952), Würzburg: Königshausen \& Neumann.

Kozlarek, Oliver. (2014a). Modernidad como conciencia del mundo. Ideas en torno a una teoría social humanista para la modernidad global, Ciudad de México, D.F.: Siglo XXI.

Kozlarek, Oliver (Ed.). (2014b). Multiple Experiences of Modernity. Toward a Humanist Critique of Modernity, Göttingen: V\&R unipress.

Larraín, Jorge. (2000). Identity and Modernity in Latin America, Cambridge et al.: Polity.

Lerner, Daniel. (1958). The passing of traditional society/Modernizing the Middle East, Glencoe-Londres, The Free Press of Glencoe-Collier Macmillan, 1958.

Miller, Nicola. (2008). Reinventing Modernity in Latin America. Intellectuals Imagine the Future: 1900-1930, New York: Palgrave MacMillan.

Moebius, Stephan. (2006). Die Zauberlehrlinge. Soziologiegeschichte des Collège de Sociologie (1937-1939), Konstanz: UVK-Verlagsgesellschaft.

Paz, Octavio. (1971). Los signos en rotación y otros ensayos, Madrid: Alianza.

Paz, Octavio. (1990). Pequeña crónica de grandes días, Ciudad de México: Fondo de Cultura Económica.

Paz, Octavio. (1993). Itinerario, Ciudad de México: Fondo de Cultura Económica. 
Paz, Octavio. (1994a). "Unidad, modernidad, tradición”, en: Octavio Paz, Obras Completas 3: Fundación y disidencia. Dominio hispánico, Ciudad de México: Fondo de Cultura Económica: 1522.

Paz, Octavio. (1994b). "Fábula de la piedra", en: Ders., Obras Completas 2: Excursiones/Incursiones. Dominio extranjero, Ciudad de México: Fondo de Cultura Económica: 469-470.

Paz, Octavio. (1996). "Claude Lévi-Strauss o el nuevo festín de Esopo", en: Octavio Paz, Obras Completas 10: Ideas y costumbre II. Usos y símbolos, Ciudad de México: Fondo de Cultura Económica: 489-563.

Paz, Octavio. (1998). El laberinto de la soledad, Madrid: Cátedra.

Pocock, J. G. A. (1987). "Modernity and Anti-modernity in the Anglophone Political Traditions" en: Shmuel N. Eisenstadt (Ed.) 1987: Patterns of Modernity, Vol. 1, The West, London: Pinter.

Pozas Horcasitas, Ricardo. (2009). "La modernidad de los modernizadores", en: Anthony Stanton (Ed.) 2009: Octavio Paz: Entre poética y política, Ciudad de México: El Colegio de México, 235-293.

Ramos, Samuel. (1999). El perfil del hombre y la cultura en México, México, Espasa Calpe/Colección Austral.

Schmidt, Volker H. (2014). Global Sociology. A Conceptual Sketch, London/ New York: Palgrave.

Stanton, Anthony. (2015). El río reflexivo. Poesía y ensayo en Octavio Paz (1931-1958), Ciudad de México: Fondo de Cultura Económica.

Taylor, Charles. (2004). Modern social imaginaries, Durham-Londres: Duke University Press.

Wagner, Peter. (2001). Theorizing modernity/Inescapability and attainability in social theory, Londres-Thousand Oaks-Nueva Delhi-Gateshead, Sage Publications-Atheneaum Press.

Wagner, Peter. (2008). Modernity as experience and interpretation/A new sociology of modernity, Cambridge-Malden, Polity Press.

Weinberg, Liliana. (2004). "El ,humanismo crítico' de Octavio Paz", en: Alberto Saladino García (Ed.), Humanismo mexicano del siglo XX, Tomo I, Toluca: Universidad Autónoma del Estado de México: 371-386. 